\title{
The mouse eye lens obsolescence (Elo) mutant: studies on crystallin gene expression and linkage analysis between the mutant locus and the $\gamma$-crystallin genes
}

\author{
Paul Quinlan, ${ }^{1,2}$ Sen-ichi Oda, ${ }^{3}$ Martin L. Breitman, ${ }^{2,4}$ and Lap-Chee Tsui ${ }^{1,2}$
}

\begin{abstract}
'Department of Genetics, Research Institute, The Hospital for Sick Children, Toronto, Ontario, Canada ${ }^{2}$ Department of Medical Genetics and Medical Biophysics, University of Toronto, Toronto, Ontario, Canada; ${ }^{3}$ Research Institute of Environmental Medicine, Nagoya University, Nagoya, Japan; ${ }^{4}$ Division of Cancer Research, Mount Sinai Hospital, Toronto, Ontario, Canada
\end{abstract}

\begin{abstract}
Previous studies showed that the mouse Elo (eye lens obsolescence) mutation is located on chromosome 1, at a site near the Len-1 locus, which is defined by a set of polymorphic $\gamma$-crystallin proteins. To investigate further the relationship between Elo and the $\gamma$-crystallins, we have examined the steady-state levels of $\gamma$-crystallin transcripts in normal and mutant eyes and analyzed the linkage relationship between the Elo locus and the $\gamma$ crystallin genes. Our data showed that, while $\gamma$-crystallin mRNA levels are preferentially reduced in the mutant eyes, the mutation does not seem to map within the $\gamma$-crystallin gene cluster. The distance between Elo and the $\gamma 6$ gene (the most proximal $\gamma$-crystallin gene member) is estimated to be $1.4 \pm 0.9 \mathrm{cM}$, whereas that between $\gamma 6$ and the distantly linked $\gamma 2$ gene is $2.7 \pm 1.3 \mathrm{cM}$. Our data also suggest the possibility of recombination hot spots within the $\gamma$-crystallin gene cluster.
\end{abstract}

[Key Words: Elo; lens development; $\gamma$-crystallin; genetic linkage]

Received June 19, 1987; accepted with revisions July 2, 1987.

Eye lens obsolescence $(E I O)$ is a fully penetrant, autosomal dominant eye disorder in mice (Oda et al. $1980 \mathrm{a}, \mathrm{b})$. The lens defect is first detectable at 12 days of gestation, when the primary fiber cells of the mutant fail to elongate properly. The secondary fiber cells also develop abnormally, leading to a general deformation of the lens and microphthalmia noticeable at birth. No other ocular or nonocular tissue appears to be affected in the mutant mice (Oda et al. 1980b).

The Elo mutation (locus) has been mapped to mouse chromosome 1 (Oda et al. 1980b), at a position immediately adjacent to the Len-1 locus (Skow 1982), which is defined by a set of polymorphic major lens proteins known as the $\gamma$-crystallins (Papaconstantinou 1967). However, the linkage relationship between the Elo and Len-1 has yet to be determined.

There are probably six $\gamma$-crystallin protein species in the mouse lens (Garber and Gold 1982). Together with the $\alpha$ - and $\beta$-crystallins, these lens-specific proteins constitute over $90 \%$ of the water-soluble fraction of the lens (Bloemendal 1977; Piatigorsky 1981). Qualitative and quantitative changes in crystallins have been detected in a variety of cataract conditions (Harding and Dilley 1976), but most of these are probably not direct results of mutations in the crystallin genes (Gelatt and Das 1984).

Six closely related mouse $\gamma$-crystallin genes have been identified (Lok et al. 1984; see below). Four cDNAs have been isolated (Shinohara et al. 1982) and sequenced (Breitman et al. 1984), and two of the genes have been studied in detail (Lok et al. 1984, 1985). Nucleotide sequence analysis revealed that the $\gamma$-crystallins are highly conserved in evolution (Moormann et al. 1982; Tomarev, et al. 1982; Breitman et al. 1984; Meakin et al. 1985). Each gene contains a small $5^{\prime}$ exon encoding only three amino acids, followed by two larger exons of similar sizes, encoding the rest of the 174-175 residues of the polypeptide (Moormann et al. 1983; Lok et al. 1984; Meakin et al. 1985). The multiple members of the $\gamma$ crystallin gene family are tandemly arranged in a cluster of rats and humans (Meakin et al. 1985; Moormann et al. 1985). It is assumed that the mouse $\gamma$-crystallin genes are similarly arranged (see below).

Individual $\gamma$-crystallin genes are differentially regulated during lens development (Harding and Dilling 1976; McAvoy 1978; van Leen et al. 1987; Murer-Orlando et al. 1987). Synthesis of $\gamma$-crystallin is restricted to the terminally differentiated lens fiber cells, whereas the $\alpha$ - and $\beta$-crystallin proteins can be found in the less- 
differentiated lens epithelium and elongating fiber cells, respectively (McAvoy 1978; Zwaan and Silver 1983). Although the onset of $\gamma$-crystallin synthesis appears to coincide with the time when the Elo phenotype is first detectable in the mutant lens (Oda et al. 1980a), immunohistochemical studies showed that necrotic process in the lens of the Elo mice was probably not due to defects in $\gamma$-crystallin synthesis (Watanabe et al. 1980).

To investigate the etiology of Elo, we have performed molecular and genetic analysis of mutant mice using DNA probes derived from five of the mouse $\gamma$-crystallin genes. In this study, we have analyzed the steady-state levels of $\gamma$-crystallin transcripts in the mutant mice and the linkage relationship between the Elo locus and the $\gamma$-crystallin genes. The latter has been accomplished through the use of restriction fragment length variants (RFLVs) associated with the mouse $\gamma$-crystallin genes. Our results show that, while $\gamma$-crystallin transcripts are drastically reduced in the mutant lenses, the Elo locus is clearly separable from the $\gamma$-crystallin genes.

\section{Results}

RNA analysis

Previous immunohistochemical studies revealed that the onset of $\gamma$-crystallin synthesis was unaffected in the lenses of Elo mice (Watanabe et al. 1980). However, because of the general disruption of lens fiber cells in the mutant lenses, it was difficult to determine whether there was any quantitative change in $\gamma$-crystallin synthesis by the antibody staining technique. To readdress this question, the levels of $\alpha-, \beta-$, and $\gamma$-crystallin tran- scripts in normal and mutant lenses were examined. Total RNAs were extracted from the eyes of normal and mutant mice and analyzed by gel-blot hybridization with radioactively labeled probes. As shown in Figure $1 \mathrm{a}$, there is a significant reduction of the steady-state level of $\gamma$-crystallin mRNA in the Elo mutant as compared to that in the normal. A careful quantitative analysis revealed that this reduction is greater than 50 -fold whereas the levels of $\alpha$ - and $\beta$-crystallin mRNAs in the Elo eyes are only decreased to approximately one-fifth of that found in normal eye lenses (Fig. 2b). Similar results were obtained when different $\gamma$-crystallin probes were used (data not shown). These data, therefore, suggest that the Elo mutation specifically affects the expression of all $\gamma$-crystallin genes.

\section{Identification of RFLVs associated with $\gamma$-crystallin genes}

Although previous studies suggested that Elo and the $\gamma$ crystallin genes might be closely linked to each other (Skow 1982), a linkage between the two loci had yet to be demonstrated. In view of the specific effect of Elo on the expression of the $\gamma$-crystallin genes, it was of immediate interest to investigate the relationship between them. However, to facilitate the analysis, it was first necessary to identify RFLVs associated with each of the five $\gamma$-crystallin gene probes (Fig. 2).

DNA from six mice was digested with 13 different enzymes and analyzed with the five $\gamma$-crystallin probes. The result of this survey showed that the mouse $\gamma$-crystallin locus is highly polymorphic among different mouse strains, as previously noted (Lok et al. 1984). For

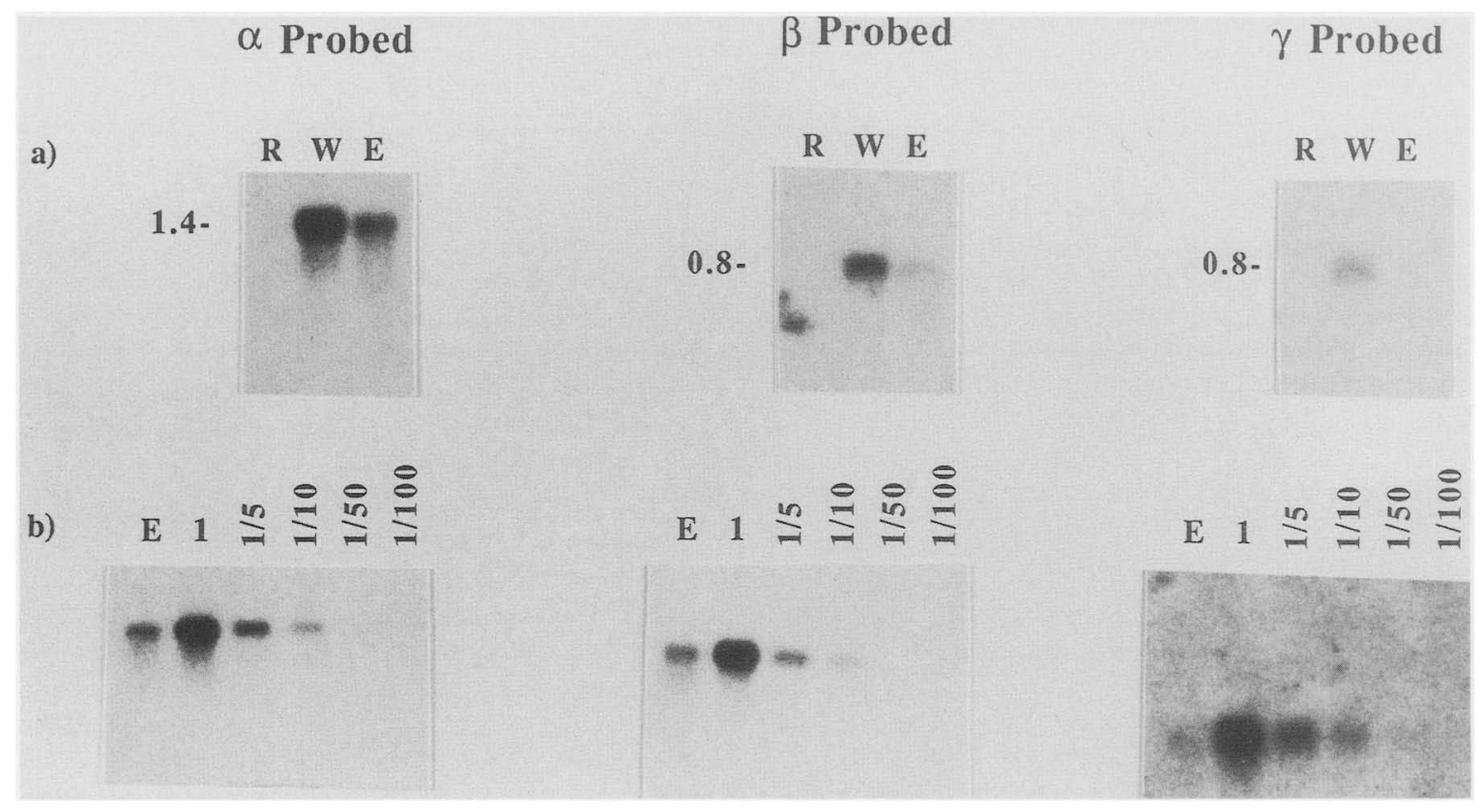

Figure 1. Analysis of crystallin mRNAs in eyes from wild-type and Elo mice. $(a)$ Each lane contains equal eye equivalents of total RNA isolated from eyes of wild type (W) or Elo (E) mice and hybridized with each of the $\alpha, \beta$, and $\gamma 2$ probes. Similar $\gamma$-crystallin mRNA hybridization results were obtained with the $\gamma 1, \gamma 3$, and $\gamma 4$ probes (data not shown). (R) E. coli rRNA marker. (b) Estimation of relative levels of crystallin mRNAs in wild-type and Elo eyes. The wild-type RNA sample was diluted by various dilution factors as indicated. 


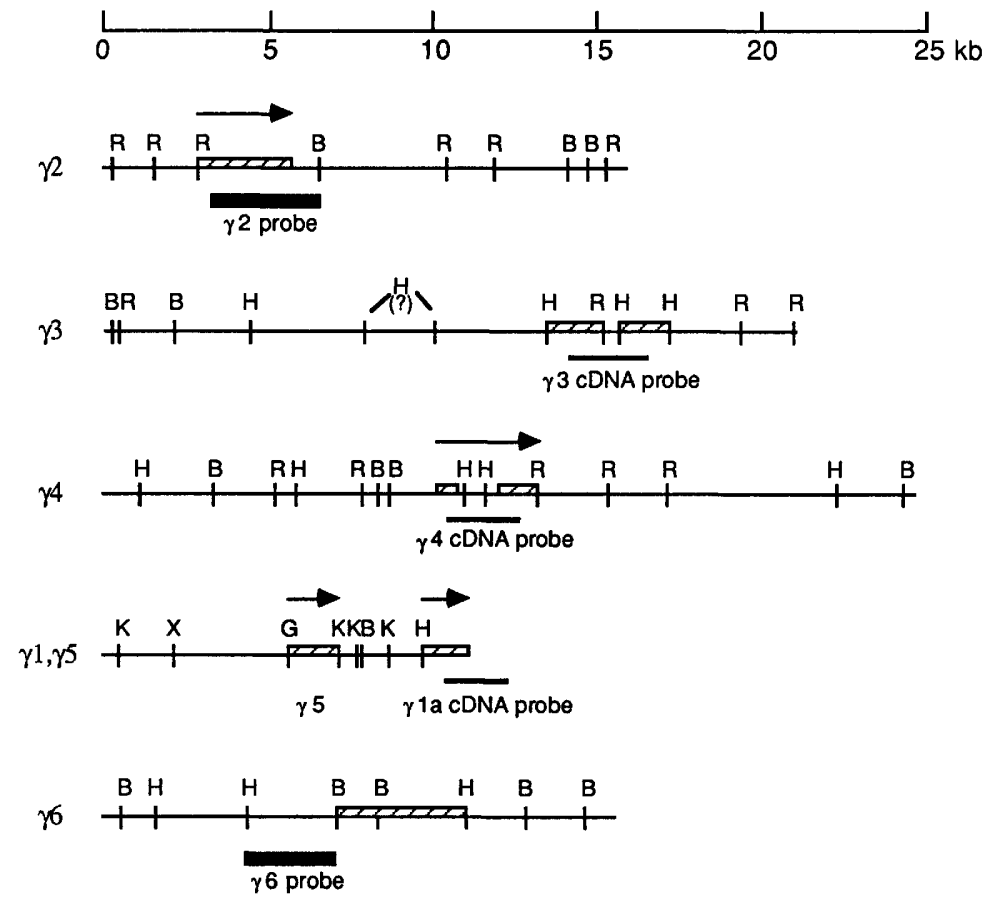

\begin{abstract}
Figure 2. Restriction maps of DNA segments containing six $\gamma$-crystallin genes. The gene assignments for $\gamma 1, \gamma 2, \gamma 3$, and $\gamma 4$ were based on DNA sequence identity with previously published cDNA sequences (Breitman et al. 1984). The designations for $\gamma 5$ and $\gamma 6$ are arbitrary. All maps, except that for $\gamma 1$ and $\gamma 5$, are derived from overlapping phage clones. Portions of the maps containing the $\gamma 2$ and $\gamma 4$ genes have been published previously (Lok et al. 1984, 1985). The hatched bars correspond to cDNA hybridizing regions. The probes used for RFLV analyses are indicated below each restriction map. Arrows indicate directions of transcription as determined by DNA sequence analyses. (H) HindIII; (B) BamHI; (R) EcoRI; (K) KpnI; (G) BglII.
\end{abstract}

example, 6 out of 12 enzymes were found to reveal an RFLV specifically associated with the $\gamma 4$ probe (data not shown). Since all $\gamma$-crystallin gene members were expected to be clustered within the same locus, only those enzymes that revealed an RFLV unique to the individual gene probes were utilized in the subsequent linkage analysis (Fig. 3). The exact polymorphic restriction enzyme recognition sites have not been localized for each probe, except for those in the $\gamma 2$-crystallin gene, for which both forms (alleles) of the RFLV have been cloned (our unpublished data). Table 1 shows the list of probes, enzymes, and RFLVs used in the present study. It was possible to use the same enzyme (SstI) digestion for both the $\gamma 2$ and $\gamma 3$ probes, since the respective RFLVs were different (see Fig. 3).

The result of the above study also showed that there is no major rearrangement or deletion of the $\gamma$-crystallin genes in Elo. All of the RFLVs detected in Elo were found in at last one other normal mouse strain (data not shown).

\section{Linkage analysis}

Since the Elo phenotype (microphthalmia) is inherited as a dominant trait and RFLVs are codominant markers, it was possible to perform a simple yet efficient phaseknown double backcross to examine the linkage relationship between these loci. Accordingly, 10 mating pairs were set up and a total of 148 offspring were examined for their eye phenotype and RFLV patterns. Examples of representative offspring are shown in Figure 3 and the data are summarized in Figures 4 and $5 a$.

Of the 148 progeny analyzed, six (E2, E8, E30, W30, W62, and W66) revealed recombination between Elo and the RFLV associated with the $\gamma 2$ gene probe (Fig. 3a). Of these six, three (E8, E30, and W30) showed recombination between Elo and the RFLVs associated with the $\gamma 3$ and $\gamma 4$ probes; and three (E2, W62, and W66) showed recombination between the latter RFLV loci and $\gamma 2$ (Fig. $3 \mathrm{~b}, \mathrm{cl}$. These results, therefore, suggest that $\gamma 3$ and $\gamma 4$ are located between $\gamma 2$ and Elo.

Of the three recombinants between Elo and $\gamma 3 / \gamma 4$, two (E30 and W30) were found to be crossovers between Elo and the RFLVs associated with the $\gamma 1$ and $\gamma 6$ genes and one (E8) between these latter RFLV loci and $\gamma 3 / \gamma 4$ (Fig. 3d,e). Therefore, these latter data are consistent with the assumption that $\gamma 1$ and $\gamma 6$ map between $\gamma 3 / \gamma 4$ and Elo.

Taking all together, a tentative genetic map for the $\gamma$-crystallin genes and Elo could be constructed (Fig. 5b). Unfortunately, the relative order for $\gamma 3$ and $\gamma 4$ and that for $\gamma 1, \gamma 5$, and $\gamma 6$ could not be determined from these data since no recombination event was detected between these loci. The position of $\gamma 5$, although not directly mapped, may be inferred by its physical linkage to the $\gamma 1$ gene (see Fig. 1).

\section{Discussion}

This study reveals that the steady-state levels of the $\gamma$ crystallin mRNAs are severely reduced in the lenses of the Elo mice. Although the Elo mutation maps closely to the mouse $\gamma$-crystallin gene cluster, it does not seem to be within any of the six gene members. The recombination distance between Elo and the nearest $\gamma$-crystallin gene member, $\gamma 6$, is estimated to be $1.4 \pm 0.9 \mathrm{cM}$, which is apparently much closer than that between the two distant members in the gene cluster; the $\gamma 6$ and $\gamma 2$ genes are estimated to be $2.7 \pm 1.3 \mathrm{cM}$ apart (Fig. 5b).

Based on the DNA sequences of these mouse genes 
Quinlan et al.

a)

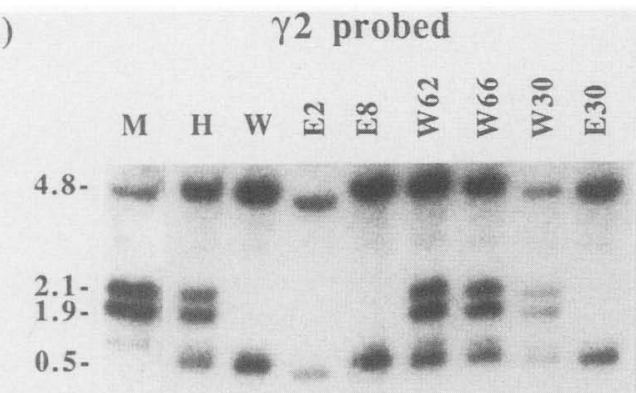

b)

$\gamma 3$ probed

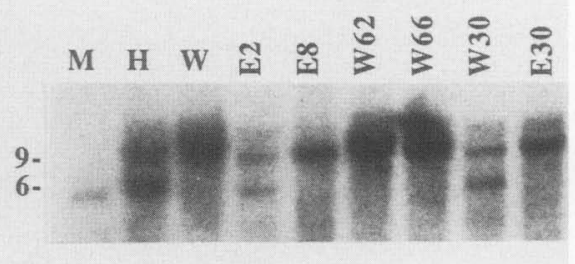

c)

$\gamma 4$ probed

d)

$\gamma 1$ probed
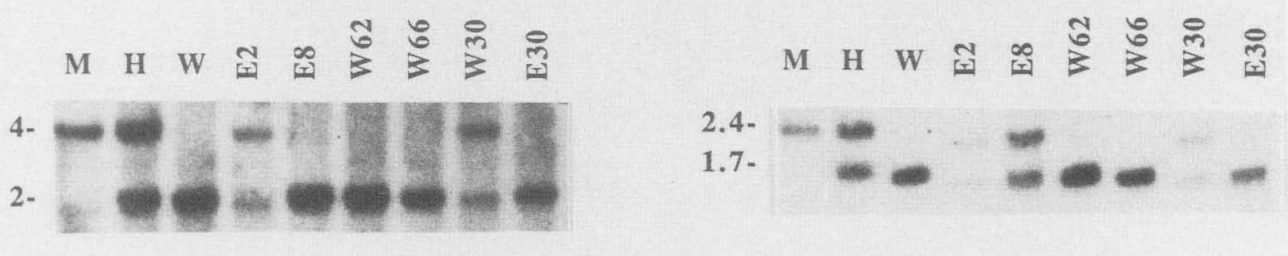

e)

$\gamma 6$ probed

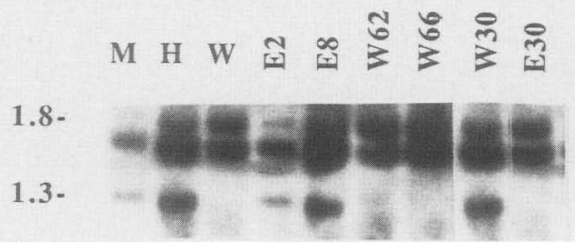

Figure 3. DNA analysis. Five micrograms of mouse DNA were digested with the indicated restriction enzymes, size-separated by electrophoresis in $0.7 \%$ agarose gels, transferred to Zetabind membranes, and hybridized with the $\gamma 2-(a), \gamma 3-(b), \gamma 4-(c), \gamma 1-(d)$, and $\gamma 6$ (e) $\gamma 6$ crystallin probes. (M) Homozygous Elo; (W) normal; (H) heterozygous Elo. E2, E8, E30, W30, W62, W66 are progeny from backcross matings [pedigree details shown in Fig. 4; (E) mice with the Elo eye phenotype; (W) mice with wild-type eye phenotype].

(Lok et al. 1984, 1985, and our unpublished data) and on sequence alignment with the orthologous members in the rat (den Dunnen et al. 1986), comparative maps of the two species could be derived (Fig. 6). The order of the

Table 1. Restriction fragment length variants (RFLVS) associated with the mouse $\gamma$-crystallin genes

\begin{tabular}{lcl}
\hline Probe & Enzyme & $\begin{array}{l}\text { Alleles } \\
(\mathrm{kb})\end{array}$ \\
\hline$\gamma 1$ & BgIII & $(1) 1.7$ \\
& & $(2) 2.4$ \\
$\gamma 2$ & SstI & $(1) 4.8+0.5$ \\
& & $(2) 2.1+1.9$ \\
$\gamma 3$ & SstI & $(1) 9.0$ \\
$\gamma 4$ & & $(2) 6.0$ \\
$\gamma 6$ & TaqI & $(1) 2.0$ \\
& & $(2) 4.0$ \\
& RsaI & $(1) 1.8$ \\
& & $(2) 1.3$ \\
\hline
\end{tabular}

${ }^{a}$ Restriction endonuclease used to reveal the RFLVs.

b Allele 1 in each case is arbitrarily assigned to the allele present on the parental chromosome carrying the wild-type gene at the Elo locus, and allele 2 to that carrying the mutant gene. mouse $\gamma$-crystallin genes derived from our linkage analysis is in good agreement with that predicted from the rat gene cluster. Our linkage data also provide information about the possible location of the rat $\gamma 4-1$ gene, which had not been physically linked to the other five gene members that were found clustered within a $50-\mathrm{kb}$ DNA segment (Moormann et al. 1985; J.G.G. Shoenmakers, pers. comm.). The comparative map suggests that the rat $\gamma 4-1$ gene is the first gene in the cluster. Furthermore, since five of the six members in the rat $\gamma$-crystallin gene family were found to be linked in a head-to-tail tandem array (Moormann et al. 1985), it is likely that the mouse genes are also similarly arranged. This arrangement would suggest that Elo is located downstream from the RFLV associated with the mouse $\gamma 6$-crystallin gene.

In view of the size of the $\gamma$-crystallin gene cluster in the rat, it is surprising to observe the high frequency of recombination among the mouse $\gamma$-crystallin genes. The physical size of this mouse gene cluster has not been established. Based on the total DNA fragment size of our gene isolates, it is probable that the cluster spans $\geqq 80 \mathrm{~kb}$ (Fig. 1). Assuming a direct correlation between the physical and genetic distance in the mouse, $1 \mathrm{cM}$ corresponds to approximately 2000 kilobases $(\mathrm{kb})$ of mouse DNA. 

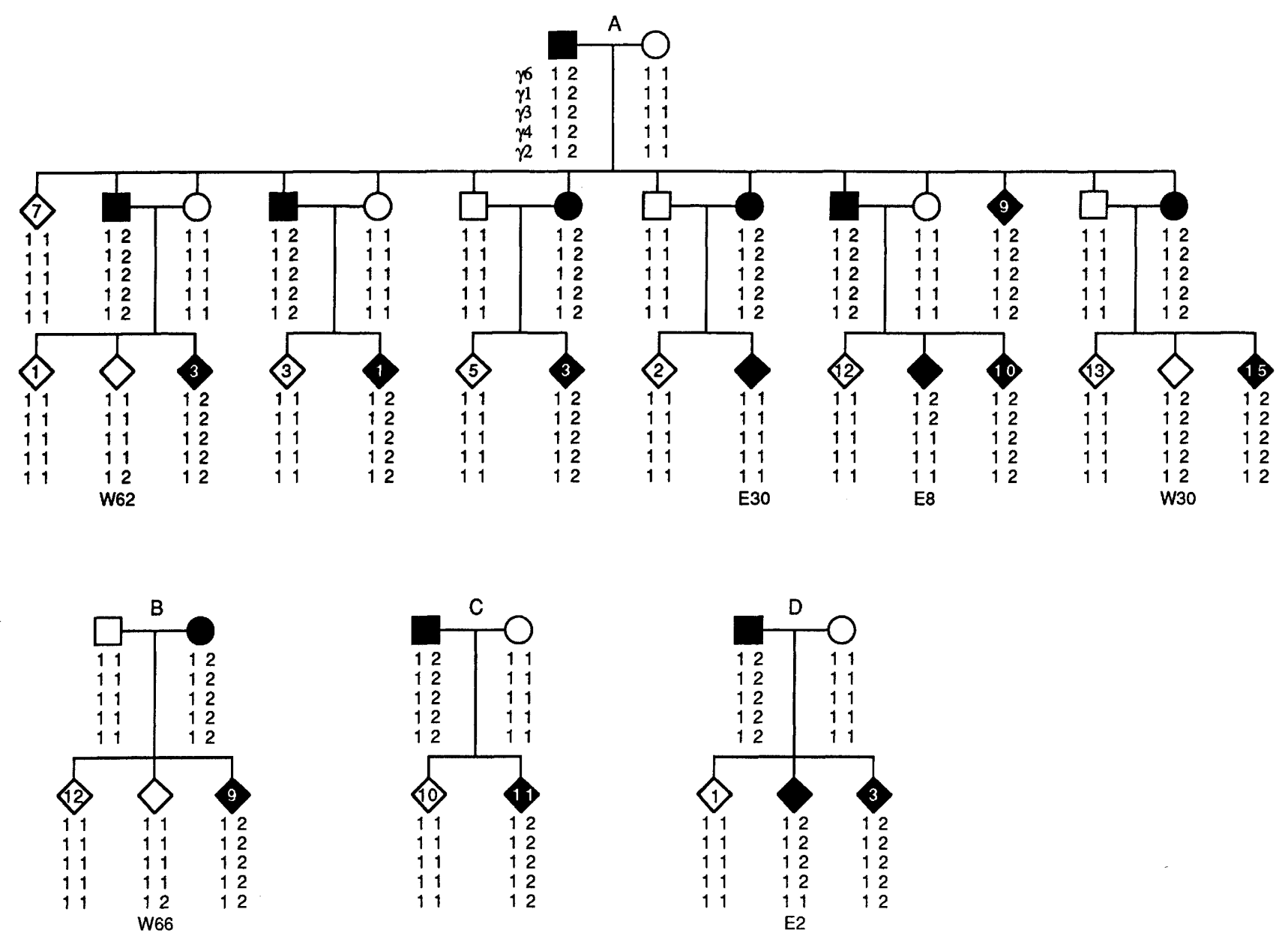

Figure 4. Pedigree data. Filled symbols denote mice with the Elo eye phenotype; open symbols denote wild type (squares $=$ males, circles $=$ females; diamonds $=$ either sex). The enclosed numbers represent numbers of mice with same phenotype. E2, E8, E30, W30, W62, W66 are recombinants (see Fig. 3). The allelic assignments for each RFLV locus are indicated below each mouse/category; their definitions are given in Table 1 . Total mice analyzed is 148.

The mouse $\gamma$-crystallin gene cluster may therefore extend over $4000 \mathrm{~kb}$ in size. This estimate is, however, heavily biased by the high frequency of recombination events $(3 / 148)$ detected between $\gamma 2$ and $\gamma 4$. The single crossover (1/148) observed between $\gamma 3$ and $\gamma 1$ might not be by itself significant.

Alternatively, recombination frequency may be much elevated within the $\gamma$-crystallin gene cluster. Recombination hot spots within mammalian genomes have been described previously (Chakravarti et al. 1984; Steinmetz et al. 1986). It is, therefore, important to determine the exact extent of the mouse $\gamma$-crystallin cluster. Longrange restriction mapped by pulsed-field gel electrophoresis (Carle and Olson 1984; Schwartz and Cantor 1984) may prove to be particularly useful for this purpose. A complete physical map of the $\gamma$-crystallin gene cluster may also provide additional insight towards cloning of the Elo mutation. Our present genetic data suggest that Elo is $1.4 \pm 0.9 \mathrm{cM}$, or $2800 \pm 1800 \mathrm{~kb}$, downstream of the mouse $\gamma 6$-crystallin gene.

The results of our RNA analysis also support the assumption that Elo is not caused by mutation in any of the $\gamma$-crystallin genes, as the steady-state levels of all $\gamma$ - crystallin transcripts are markedly reduced in the mutant mice. Although we have not examined the RNA levels in homozygous Elo mice, due to difficulties in maintaining a homozygous breeding stock, no difference was detectable in the eye morphology between Elo/ + and Elo/Elo mice. Therefore, Elo is probably a mutation that specifically affects the differentiation of the lens fiber cells where the $\gamma$-crystallin genes are expressed. Furthermore, since the levels of both $\alpha$ - and $\beta$-crystallin mRNAs are apparently not as severely affected as the $\gamma$-transcripts, it is possible that the Elo mutation acts at a point after the commencement of $\alpha$ - and $\beta$-crystallin synthesis but before or during the period of $\gamma$-crystallin gene expression.

A number of lens growth factors have been reported in the literature (Reddan and Wilson-Dziedzic 1983; Campbell and McAvoy 1986; Moenner et al. 1986). The fully penetrant, dominant phenotype of Elo makes it unlikely that the lens defect in Elo is due to mutation in any of the positively regulating growth factors or growth factor receptors. For the same reason, it is unlikely that the Elo mutation lies in one of the structural components of the lens. However, it is possible that this muta- 
Quinlan et al.

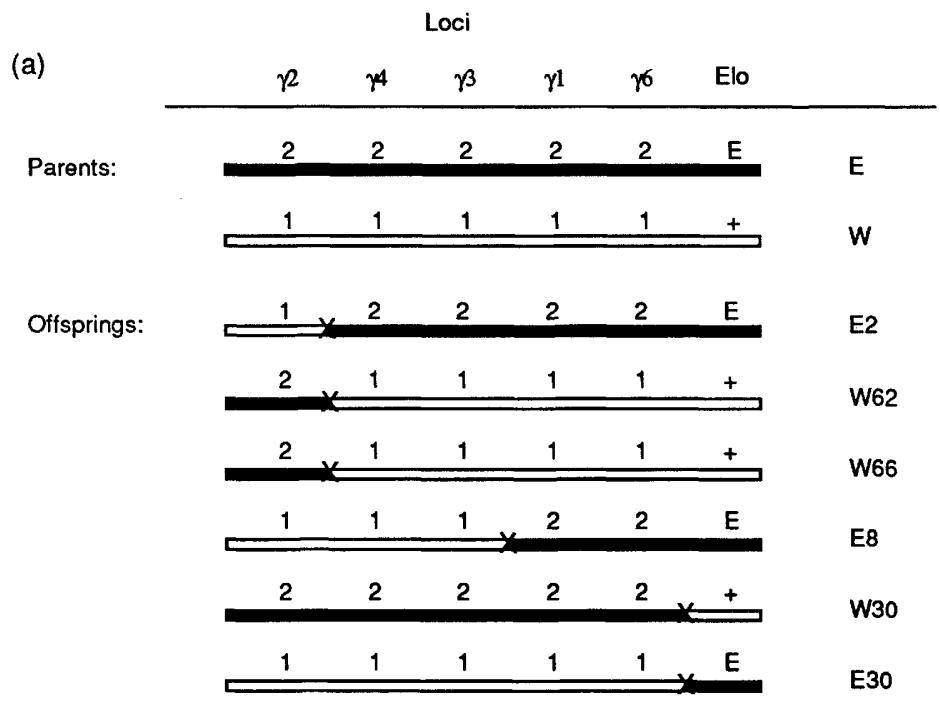

Figure 5. Summary of recombinant events. $(a)$ The parental and the recombinant genotypes. (W) Wild-type parental chromosome; (E) Elo parental chromosome. E2, E8, and E30 are recombinant chromosomes in mice with the Elo eye phenotype; W30, W62, and W66 are recombinant chromosomes from mice with wild-type eye phenotype. The putative crossover points are indicated by X. (b) Genetic distance estimates between Elo and the $\gamma$-crystallin marker loci.

(b)

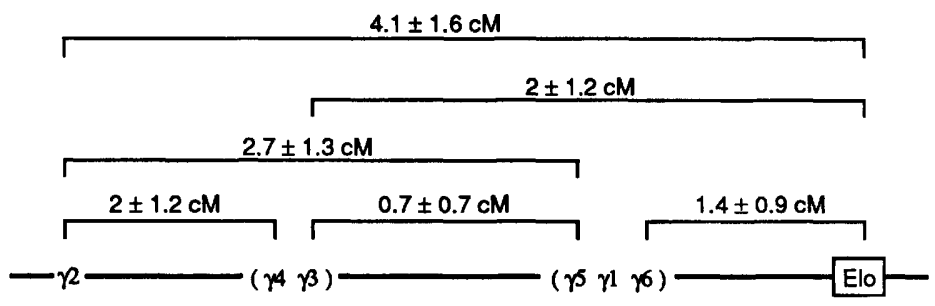

tion is caused by the presence of a factor that inhibits lens fiber cell elongation. Such factors have been implicated in normal lens development where the formation of fiber cells may be inhibited by factors present in the aqueous humor (Reddan 1982). In the case of Elo, an unscheduled expression of one of these putative /negative regulatory) factors may lead to the blockage of fiber cell formation.

The precise localization of the mutant and cloning of the gene should permit a direct identification of the gene defect in Elo and provide new insight into the regulation of lens development.

\section{Materials and methods}

\section{Mouse strains}

The Elo mouse arose from a spontaneous mutation during brother-sister matings between C57BL/6JNga and Japanesefancy mouse strains. It was subsequently maintained in a $\mathrm{C} 3 \mathrm{Hf} / \mathrm{HeNem}$ background (Oda et al. 1980a). Due to recent difficulties in propagating the homozygous Elo mice in the $\mathrm{C} 3 \mathrm{H}$ background, the mutant has been out-crossed with the Slc : ICR strain and maintained by backcross and occasional intercross matings. Mice heterozygous or homozygous for the Elo mutation cannot be distinguished by pathology of the eyes, and microphthalmia correlates with lens degeneration in all cases examined.

Mice were sacrificed for RNA and DNA analysis by cervical dislocation. Mice at 8 days of age were used for eye RNA prepa- ration and those at approximately 30 days were used for DNA analysis.

\section{RNA and DNA isolation}

For RNA extraction, normal and mutant eyes were removed surgically and immediately frozen in liquid nitrogen. Frozen tissue samples (10 eyes) were resuspended in $4 \mathrm{ml}$ of guanidinium isothiocyanate and homogenized for $1 \mathrm{~min}$ with a Kinematica Polytron. Each homogenate was layered over a $3-\mathrm{ml}$ cushion of $5.7 \mathrm{M} \mathrm{CsCl}$ and centrifuged at $20^{\circ} \mathrm{C}$ in a Beckman SW41 rotor at $30,000 \mathrm{rpm}$ for $16 \mathrm{hr}$. The RNA pellets were each dissolved in $0.4 \mathrm{ml}$ of diethylpyrocarbonate (DEP)-treated water and extracted twice with equal volumes of $\mathrm{pH}$-buffered phenol and chloroform. The RNA samples were then precipitated in $75 \%$ ethanol and each redissolved in $0.4 \mathrm{ml}$ of $\mathrm{DEP}-\mathrm{H}_{2} \mathrm{O}$ ready for gel-blot hybridization analysis.

Mouse genomic DNAs were isolated from spleens. Individual spleens were each lysed in $5 \mathrm{ml}$ of $10 \mathrm{~mm}$ Tris- $\mathrm{HCl} / 10 \mathrm{~mm}$ EDTA/1\% SDS (pH 7.5) and treated overnight with $50 \mu \mathrm{g} / \mathrm{ml}$ self-digested Pronase. The samples were then phenol-extracted and DNA isolated essentially as described previously (Lok et al. 1984).

\section{Gene probes}

Three $\gamma$-crystallin cDNAs and two genomic DNA clones were used as probes for DNA hybridization analyses. All of these probes specifically detect their corresponding gene sequences under the conditions used in this study. pM $\gamma \mathrm{la}$ is a cDNA clone containing sequences from exons 1 and 2 as well as a 


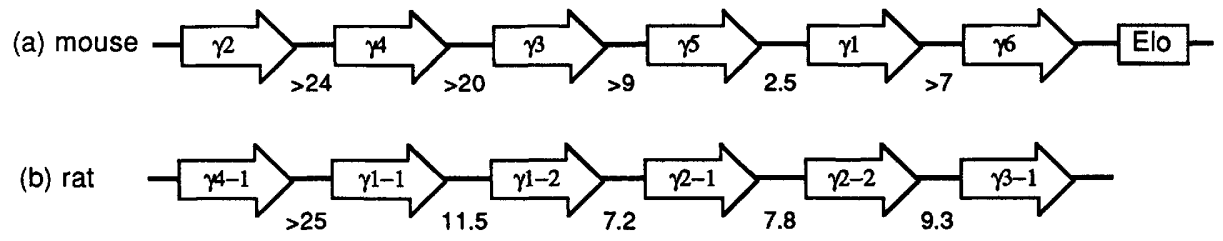

Figure 6. Physical organizations of the mouse $|a|$ and rat $|b| \gamma$-crystallin gene clusters. Arrows indicate direction of transcription determined by DNA sequence analysis (Moormann et al. 1985; our unpublished data), except those for the mouse $\gamma 2$, $\gamma 4, \gamma 3$, and $\gamma 6$, and the rat $\gamma 4-1$ which are presumptive (see text for details). Intergenic distance estimates are shown in kilobases.

portion of intron 2 of the mouse $\gamma 1$-crystallin gene (Shinohara et al. 1982; Breitman et al. 1984). pM $\gamma 3$ and $\mathrm{pM} \gamma 4$ are partial cDNA clones for the $\gamma 3$ and $\gamma 4$ genes (Shinohara et al. 1982; Breitman et al. 1984; Lok et al. 1984). The $\gamma 2$ gene-specific probe is a $3.2-\mathrm{kb} N c o I-B a m H I$ fragment from the mouse $\gamma 2$ crystallin gene (Lok et al. 1985). Although additional hybridizing bands were observed with this probe in some enzyme digestions, the $\gamma 2$ specific bands were clearly distinguishable from others based on signal intensity and the $\gamma 2$ restriction map. The fifth gene-specific probe is a $2.8-\mathrm{kb}$ HindIII-BamHI fragment isolated from the $\gamma 6$ gene clone (see Fig. 1).

The $\alpha$ - and $\beta$-crystallin cDNA clones, pM $\alpha \mathrm{ACrl}$ and pM $\beta C r 1$, have been described previously (Inana et al. 1982; King et al. 1982). The characterizations of the $\gamma 2$ and $\gamma 4$ genes have been described previously (Lok et al. 1984, 1985). Description of the cloning of the $\gamma 1, \gamma 3, \gamma 5$, and $\gamma 6$ genes will be presented elsewhere.

\section{Gel-blot hybridization analysis}

RNA samples ( $3 \mu \mathrm{g}$ each) were size-fractionated by electrophoresis on formaldehyde-agarose gels and transferred to nitrocellulose filters (Schleicher \& Schuell BA85). The conditions for filter prehybridization and hybridization were essentially as described (Murer-Orlando et al. 1987).

DNA samples $(5-10 \mu \mathrm{g}$ each) were digested with a variety of restriction endonucleases (Bohringer/Mannheim and New England BioLabs) and size-fractionated by electrophoresis in $0.7 \%$ agarose (Seakem) with Tris-acetate buffer as described previously (Lok et al. 1984; Meakin et al. 1985). The samples were then transferred to Zetabind membrane filters (AMF-Cuno), prehybridized, and hybridized as described by the supplier.

Radioactive ${ }^{32} \mathrm{P}$-labeled DNA probes (sp. act. $\geqq 3 \times 10^{8} \mathrm{cpm} /$ $\mu \mathrm{g}$ ) were prepared by using the random-priming method (Feinberg and Vogelstein 1983). After hybridization, the filters were washed twice at room temperature with $2 \times \mathrm{SSC} / 0.1 \%$ SDS $\mid 1 \times \mathrm{SSC}=150 \mathrm{mM} \mathrm{NaCl}$ and $15 \mathrm{~mm}$ sodium citrate $\mid$ followed by four washes in $0.1 \times \mathrm{SSC} / 0.1 \% \mathrm{SDS}$ at $65^{\circ} \mathrm{C}$. The filters were then air-dried and exposed to Kodak XAR- 5 films with Cronex Lightening-Plus intensifying screens at $-70^{\circ} \mathrm{C}$ for $12 \mathrm{hr}$ to 1 week.

\section{Survey of RFLVS}

DNAs from six mice (two Elo homozygotes, two Elo heterozygotes, two wild-type homozygotes) were digested with a panel of 13 different commonly used restriction enzymes (ApaI, BamHI, BglI, BglII, EcoRI, HindIII, MspI, PstI, PvuII, RsaI, SstI, TaqI, XbaI) and examined for variations in the sizes of DNA fragments hybridized to the five gene probes. The enzymes revealing an RFLV unique to the individual probes were chosen for the subsequent linkage analysis.

\section{Linkage analysis}

To analyze the linkage relationship between Elo and the $\gamma$-crystallin genes, matings were set up between heterozygous Elo $(E l o /+)$ and homozygous wild-type $(+/+)$ mice. The parental genotypes of the RFLVs associated with the $\gamma$-crystallin genes were assumed to be heterozygous for the Elo and homozygous for the wild-type mice; they were later confirmed by DNA analysis. Offspring from these double backcross matings were examined for their eye morphology (presence or absence of microphthalmia) and the RFLVs associated with the $\gamma$-crystallin genes. Recombination events between Elo and the RFLV loci were easily identified by their new marker/phenotype combinations which were different from both parents. The genetic distance was estimated by direct counting of the recombination fraction, $\theta=r / n$, where $r$ is the number of recombinants and $n$ is the total number of meioses. The standard error was calculated by using the equation: $\sigma=\sqrt{[\theta(1-\theta / n]}$ (Ott 1985).

\section{Acknowledgments}

The authors wish to thank J. Piatagorsky for providing $\alpha-, \beta-$, and $\gamma$-crystallin cDNA clones and Qijing Wang for DNA analysis on some of the mouse offspring. P.Q. is the recipient of an Ontario Graduate Scholarship. This study was supported by a grant from the University of Toronto and Medical Research Council (MRC) of Canada. M.L.B. and L.-C.T. are research scholars of the MRC and the Canadian Cystic Fibrosis Foundation, respectively.

\section{References}

Bloemendal, H. 1977. The vertebrate eye lens. Science 197: $127-137$.

Breitman, M.L., S. Lok, G. Wistow, J. Piatigorsky, J.A. Tréton, R.J.M. Gold, and L.-C. Tsui. 1984. $\gamma$-Crystallin family of the mouse lens: Structural and evolutionary relationships. Proc. Natl. Acad. Sci. 81:7762-7766.

Campbell, M.T. and J.W. McAvoy. 1986. A lens differentiation factor from calf neural retina. Exp. Cell Res. 163:453-466.

Carle, G.F. and M.V. Olson. 1984. Separation of chromosomal DNA molecules from yeast by orthogonal-field-alteration gel electrophoresis. Nucleic Acids Res. 14:5647-5664.

Chakravarti, A., K.H. Buetow, S.E. Antonarakis, P.G. Waber, C.D. Boehm, and H.H. Kazazian. 1984. Nonuniform recombination within the human $\beta$-globin gene cluster. Am. $/$. Hum. Genet. 36:1239-1258.

den Dunnen, J.T., R.J.M. Moormann, N.H. Lubsen, and J.G.G. Schoenmakers. 1986. Concerted and divergent evolution within the rat $\gamma$-crystallin gene family. J. Mol.Biol. 189: 3746.

Feinberg, A.P. and B. Vogelstein. 1983. A technique for radiola- 
beling DNA restriction endonuclease fragments to high specific activity. Anal. Biochem. 132: 6-13.

Garber, A.T. and R.J.M. Gold. 1982. Comparative two-dimensional electrophoretic analysis of water soluble proteins from bovine and murine lenses. Exp. Eye Res. 35: 585-596.

Gelatt, K.N. and N.D. Das. 1984. Animal models for inherited cataracts: A review. Curr. Eye Res. 3: 765-778.

Harding, J.J. and K.J. Dilley. 1976. Structural proteins of the mammalian lens: A review with emphasis on changes in development, aging and cataract. Exp. Eye Res. 22: 1-73.

Inana, G., T. Shinohara, J.V. Maizel, and J. Piatigorsky. 1982. Evolution and diversity of the crystallins. Nucleotide sequence of a $\beta$-crystallin mRNA from the mouse lens. J. Biol. Chem. 257: 9064-9071.

King, C.R., T. Shinohara, and J. Piatigorsky. 1982. $\alpha$ A-crystallin messenger RNA of the mouse lens: More non-coding than coding sequences. Science 215: 985-987.

Lok, S., L.-C. Tsui, T. Shinohara, J. Piatigorsky, R.J.M. Gold, and M.L. Breitman. 1984. Analysis of the mouse $\gamma$-crystallin gene family: Assignment of multiple cDNAs to discrete genomic sequences and characterization of a representative gene. Nucleic Acids Res. 12: 4517-4529.

Lok, S., M.L. Breitman, A.B. Chepelinsky, J. Piatigorsky, R.J.M. Gold, and L.-C. Tsui. 1985. Lens-specific promoter activity of a mouse $\gamma$-crystallin gene. Mol. Cell. Biol. 5: 2221-2230.

McAvoy, J.W. 1978. Cell division, cell elongation and distribution of $\alpha-, \beta-$, and $\gamma$-crystallins in the rat lens. J. Embryol. Exp. Morphol. 44: 149-165.

Meakin, S.O., M.L. Breitman, and L.-C. Tsui. 1985. Structural and evolutionary relationships among five members of the human $\gamma$-crystallin gene family. Mol. Cell. Biol. 5: 14081414.

Moenner, M., B. Chevallier, J. Badet, and D. Barritault. 1986. Evidence and characterization of the receptor to eye-derived growth factor $\mathrm{I}$, the retinal form of basic fibroblast growth factor, on bovine epithelial lens cells. Proc. Natl. Acad. Sci. 83: 5024-5028.

Moormann, R.J.M., J.T. den Dunnen, H. Bloemendal, and J.G.G. Schoenmakers. 1982. Extensive intragenic sequence homology in two distinct rat lens $\gamma$-crystallin cDNAs suggests duplication of a primordial gene. Proc. Natl. Acad. Sci. 79: 6876-6880.

Moormann, R.J.M., J.T. den Dunnen, L. Mulleners, P.M. Andreoli, H. Bloemendal, and J.G.G. Schoenmakers. 1983. Strict co-linearity of genetic and protein domains in an intragenically duplicated rat lens $\gamma$-crystallin gene. J. Mol. Biol. 171: 353-368.

Moormann, R.J.M., J.T. den Dunnen, J. Heuyerions, R.J.E. Jongbloed, R.W. van Leen, N.H. Lubsen, and J.G.G. Schoenmakers. 1985. Characterization of the rat $\gamma$-crystallin gene family and its expression in the eye lens. I. Mol. Biol. 182: $419-430$.

Murer-Orlando, M., R.C. Paterson, S. Lok, L.-C. Tsui, and M.L. Breitman. 1987. Differential regulation of $\gamma$-crystallin genes during mouse lens development. Dev. Biol. 119: 260-267.

Oda, S.-I., K. Watanabe, H. Fujisawa, and Y. Kameyama. 1980a. Impaired development of lens fibers in the genetic microphthalmia, eye lens obsolescence, Elo, of the mouse. Exp. Eye Res. 31: 673-681.

Oda, S.-I., K. Watanabe, and K. Kondo. 1980b. A new mutation, eye lens obsolescence, Elo on chromosome 1 in the mouse. Ipn. J. Genet. 55: 71-75.

Ott, J. 1985. Analysis of human genetic linkage. Johns Hopkins University Press, Baltimore.

Papaconstantinou, J. 1967. Molecular aspects of lens cell differentiation. Science 156: 338-446.

Piatigorsky, J. 1981. Lens differentiation invertebrates. A re- view of cellular and molecular features. Differentiation 19: $134-153$.

1984. Lens crystallins and their gene families. Cell 38: $620-621$.

1987. Gene expression and genetic engineering in the lens. Invest. Opthal. Vis. Sci. 28: 9-28.

Reddan, J.R. 1982. Control of cell division in the ocular lens, retina, and vitreious humor. In Cell biology of the eye (ed. D.S. McDevitt), pp. 299-375. Academic Press, New York.

Redden, J.R. and D. Wilson-Dziedzic. 1983. Insulin growth factor and epidermial growth factor trigger mitosis in lenses cultured in a serum-free medium. Invest. Opthal. Vis. Sci. 24: 409-416.

Schwartz, D.C. and C.R. Cantor. 1984. Separation of yeast chromosome-sized DNAs by pulsed field gradient gel electrophoresis. Cell 37: 67-75.

Shinohara, T., E. Robinson, E. Appella, and J. Piatigorsky. 1982. Multiple $\gamma$-crystallins of the mouse lens: Fractionation of mRNAs by cDNA cloning. Proc. Natl. Acad. Sci. 79: 27832787.

Skow, L. 1982. Location of a gene controlling electrophoretic variation in mouse $\gamma$-crystallins. Exp. Eye Res. 34: 509-516.

Steinmetz, M., D. Stephan, and K.F. Lindahl. 1986. Gene organization and recombinational hotspots in the murine major histocompatibility complex. Cell 44: 895-904.

Tomarev, S.I., A.S. Krayev, K.G. Skryabin, A.A. Bayev, and G.G. Gause, Jr. 1982. The nucleotide sequence of a cloned cDNA corresponding to one of the $\gamma$-crystallins from the eye lens of the frog Rana temporaria. FEBS Lett. 146: 315-318.

van Leen, R.W., K.E.P. van Roozendaal, N.H. Lubsen, and J.G.G. Schoenmakers. 1987. Differential expression of crystallin genes during development of the rat eye lens. Dev. Biol. 120: 457-464.

Watanabe, K., H. Fujisawa, S.-I. Oda, and Y. Kameyama. 1980 Organ culture and immunohistochemistry of the genetically malformed lens, in eye lens obsolenscence, Elo, of the mouse. Exp. Eye Res. 31: 683-689.

Zwaan, J. and J. Silver. 1983. Crystallin synthesis in the lens rudiment of a strain of mice with congential anophthalmia. Exp. Eye Res. 36: 551-557. 


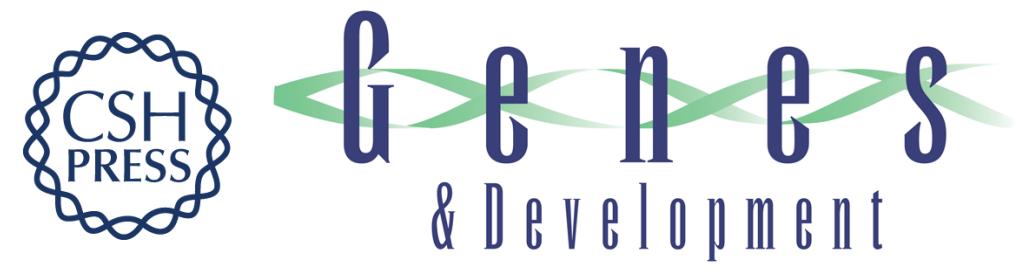

\section{The mouse eye lens obsolescence (Elo) mutant: studies on crystallin gene expression and linkage analysis between the mutant locus and the gamma-crystallin genes.}

P Quinlan, S Oda, M L Breitman, et al.

Genes Dev. 1987, 1:

Access the most recent version at doi:10.1101/gad.1.7.637

References This article cites 36 articles, 10 of which can be accessed free at:

http://genesdev.cshlp.org/content/1/7/637.full.html\#ref-list-1

License

Email Alerting Receive free email alerts when new articles cite this article - sign up in the box at the top Service right corner of the article or click here.

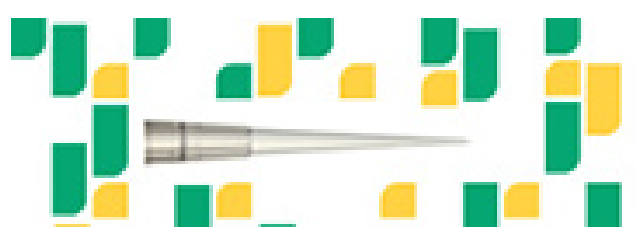

Focused on your science. 